\title{
Grouping of Metastatic Thyroid Carcinoma by Molecular Imaging Features to Allow for Individualized Treatment, with Emphasis on the TENIS Syndrome
}

\author{
Sandip Basu and Rahul Vithalrao Parghane \\ Radiation Medicine Centre, Bhabha Atomic Research Centre, Tata Memorial Hospital Annexe, Parel, Bombay, India
}

\begin{abstract}
The process of radioiodine $\left({ }^{131} I\right)$ refractoriness, dedifferentiation, and development of thyroglobulin elevation and negative iodine scintigraphy (TENIS) syndrome in metastatic differentiated thyroid carcinoma (DTC) could be viewed as a disease continuum rather than a single event or an all-or-none phenomenon. In this report, the important role of molecular functional imaging in systematically exploring the disease biology and course of metastatic DTC is presented. Making this role the base, this report proposes a novel clinical classification for TENIS syndrome using molecular functional imaging. Such an endeavor, in our view, would aid in classification into subgroups and development of an individualized treatment strategy for patients with metastatic DTC. Case illustrations are presented that substantiate this viewpoint, including the value of combined assessment in selected cases and the requirement of a multipronged treatment approach for certain case scenarios. In addition to multiple other considerations, we believe that molecular functional imaging-based characterization of metastatic lesions could evolve as an important determinant for defining the optimal treatment strategy in patients with metastatic DTC, making the clinical management more rational and scientific.
\end{abstract}

Key Words: differentiated thyroid carcinoma; thyroglobulin; radioiodine scan; ${ }^{18} \mathrm{~F}-\mathrm{FDG} \mathrm{PET} / \mathrm{CT}$; TENIS; ${ }^{68} \mathrm{Ga}$-DOTATATE

J Nucl Med Technol 2016; 44:184-189

DOI: $10.2967 /$ jnmt.116.177881

$\mathbf{T}$ he optimal treatment strategy for patients with metastatic differentiated thyroid carcinoma (DTC) encompasses multifactorial considerations such as site and size of the metastatic lesions, radioiodine $\left({ }^{131} \mathrm{I}\right)$ uptake in the lesions, tumor histology, patient characteristics, clinical status, and symptoms. The overall response and final outcome have been a function of these factors, although clinical behavior, disease course, and final outcome vary considerably between two individuals even when the anatomic features of their metastatic lesions match.

Received May 6, 2016; revision accepted Jun. 27, 2016.

For correspondence or reprints contact: Sandip Basu, Radiation Medicine Centre, Bhabha Atomic Research Centre, Tata Memorial Hospital Annexe, Jerbai Wadia Rd., Parel, Bombay 400012.

E-mail: drsanb@yahoo.com.

Published online Aug. 4, 2016.

COPYRIGHT (C) 2016 by the Society of Nuclear Medicine and Molecular Imaging, Inc.
The management of patients with thyroglobulin elevation and negative iodine scintigraphy (TENIS syndrome) has been a topic of considerable interest and debate among attending physicians and has been emphasized in recent years, particularly in view of poor outcomes, the associated challenges, and the absence of a definitive consensus on management (1-4). Management continues to evolve, with several routine and investigational therapeutic approaches being explored with respect to their place and efficacy in this challenging clinical situation, including empiric ${ }^{131}$ I therapy and whether it is justified $(1-4)$. It is imperative that with a more critical look into this entity, an individualized treatment approach be adopted, with the broad group being divided into various smaller segments based on both clinicopathologic and molecular imaging lesional characteristics.

In this present communication, we explore the potential of multitracer molecular imaging in subsegmenting the spectrum of metastatic DTC and TENIS syndrome into various subgroups and targeting an individualized management approach. This ability, in our view, would aid in creating a more rational and scientific approach for these patients. We particularly emphasize dedifferentiation and TENIS syndrome, and we propose a decisiontree type of classification and risk stratification, along with predictive personalized management strategies.

\section{METASTATIC DTC WITH SOME LESIONS DEMONSTRATING ${ }^{131}$ I UPTAKE}

DTC and TENIS syndrome have often been construed as an all-or-none phenomenon in the clinical parlance of thyroid cancer practice. However, from a theoretic standpoint and a management viewpoint, they should ideally be viewed as a disease continuum, considering the fact that dedifferentiation of metastatic DTC lesions is a progressive phenomenon that may evolve over months. Hence, in practice, a subset of metastatic DTC patients could be encountered (though not commonly) whose lesions are in this interim phase of the evolution of dedifferentiation. The first case illustration describes the disease biology and progression of such a DTC patient, in whom some metastatic lesions showed ${ }^{131}$ I uptake whereas others did not.

\section{Case 1}

A 62-y-old man with papillary thyroid carcinoma (PTC) and bilateral lung metastasis was treated with multiple 


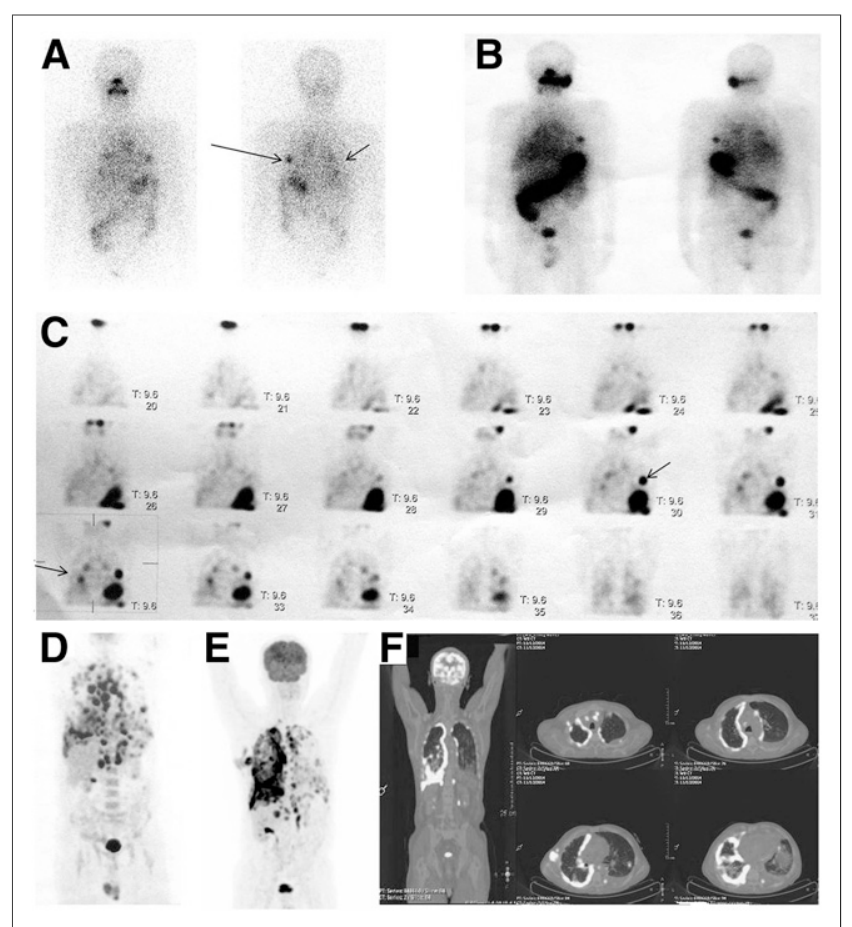

FIGURE 1. (A) Diagnostic ${ }^{131} \mid$ scan showed some ${ }^{131} \mid$-avid foci in both lungs, though lesions were much more numerous on ${ }^{18} \mathrm{~F}$ FDG PET/CT scan (D), which showed multiple metabolically active foci in both lungs and pleurae, as well as mediastinal foci. Lesions on posttreatment ${ }^{131}$ I planar scan (B) and SPECT scan (C) were similar to those on diagnostic ${ }^{131}$ I scan. Comparative ${ }^{18} \mathrm{~F}-\mathrm{FDG}$ PET/CT scan 1 y previously (D) and present scan ( $E$ and $F$ ) show progressive disease. Serum thyroglobulin level was more than $300 \mathrm{ng} / \mathrm{mL}$ on both occasions. Therapy with tyrosine kinase inhibitor (sorafenib) was started in view of progressive symptomatic disease on 18F-FDG PET/CT. Arrows indicate tracer uptake or lack thereof. A color version of $F$ is available as a supplemental file at http://tech.snmjournals.org.

doses of ${ }^{131}$ I therapy (with a cumulative dose of 17,945 $\mathrm{MBq}[485 \mathrm{mCi}]$ at the time this report was being prepared). For $2 \mathrm{y}$, large-dose ${ }^{131} \mathrm{I}$ scanning had continued to show ${ }^{131}$ I-positive lesions in both lungs. After 2 y of treatment, ${ }^{18} \mathrm{~F}$-FDG PET/CT also showed ${ }^{18} \mathrm{~F}-\mathrm{FDG}$-positive lesions in both lungs, with involvement being greater in the right lung than the left (the entire right hemithorax had multiple pleura-based nodules), and the lesions were more numerous than seen on the previous diagnostic and posttreatment ${ }^{131}$ I planar scans (Figs. 1A and 1B, respectively) or the SPECT scan (Fig. 1C). One year later, follow-up ${ }^{18} \mathrm{~F}-$ FDG PET/CT (Figs. 1E and 1F) demonstrated substantial disease progression in both lungs but more prominently in the right lung. The serum thyroglobulin level was more than $300 \mathrm{ng} / \mathrm{mL}$ on both occasions. Hence, alternative treatment modalities were sought: from the nature of the involvement, the radiation oncologist inferred that the patient was unlikely to benefit from palliative radiotherapy to the right chest wall. Treatment with the tyrosine kinase inhibitor sorafenib, $400 \mathrm{mg}$ twice daily, was considered after clinical evaluation of the patient's fitness. At 2 mo after therapy, the dose was reduced to $75 \%$ in view of hand-foot syndrome. At $5 \mathrm{mo}$, the patient is currently under observation while taking daily sorafenib.

\section{Management Rationale}

This case exemplifies the group of patients with partial dedifferentiation of metastatic lesions, in which the requirement for multimodality treatment is underscored. Although ${ }^{131} \mathrm{I}$ therapy could be one possible approach, intended primarily for ${ }^{131} \mathrm{I}$-positive lesions; the ${ }^{18} \mathrm{~F}-\mathrm{FDG}$-avid (and ${ }^{131} \mathrm{I}$-negative) metastatic lesions could behave in an aggressive fashion and be unlikely to benefit or stabilize from ${ }^{131}$ I therapy alone. Hence, ${ }^{131}$ I therapy cannot be the sole management approach in these patients, unlike patients in whom all metastatic lesions demonstrate ${ }^{131}$ I uptake. The optimal therapeutic strategy requires exploration of a multipronged approach that includes the existing routine approaches (e.g., external radiotherapy where indicated) and investigational approaches chosen on the basis of lesional characteristics (e.g., tyrosine kinase inhibitors, sorafenib, and lenvatinib or peptide receptor radionuclide therapy (PRRT) with ${ }^{177} \mathrm{Lu}$-based somatostatin receptor analogs, if adequate uptake is demonstrated in diagnostic studies with ${ }^{68} \mathrm{Ga}-$ DOTANOC or ${ }^{68} \mathrm{Ga}$-DOTATATE PET/CT).

The choice and sequence of therapies in the combined approach is an issue that needs to be explored in multidisciplinary meetings and would require prospective studies for further clarification. Administration of ${ }^{131} \mathrm{I}$ therapy before the other approaches is the usual rational choice in order to maximize the benefit of this targeted therapy while minimizing adverse effects.

\section{TENIS SYNDROME WITH INCREASING ${ }^{18} \mathrm{~F}-\mathrm{FDG}$ UPTAKE AND ${ }^{68} \mathrm{GA}$-DOTATATE AVIDITY}

\section{Case 2}

A 48-y-old woman with the follicular variant of PTC with a poorly differentiated component had undergone total thyroidectomy followed by ${ }^{131}$ I ablative therapy, with posttreatment whole-body scanning demonstrating only a neck focus. The patient presented 3 y later with neck recurrence (neck ultrasonography showing metastatic left cervical lymphadenopathy at level IV), for which she underwent nodal resection. She presented 2 y later with a rising thyroglobulin level but negative ${ }^{131} \mathrm{I}$ scintigraphy results. ${ }^{18} \mathrm{~F}-\mathrm{FDG}$ PET/CT demonstrated abnormal foci in the right lung (infrahilar region) and in the right and left axillary lymph nodes (biopsied and confirmed to be metastatic poorly differentiated thyroid carcinoma). The patient received empiric ${ }^{131} \mathrm{I}$ therapy $(7,400 \mathrm{MBq}[200 \mathrm{mCi}])$, and posttreatment scanning was negative for uptake. She was observed while on levothyroxine suppression, and followup ${ }^{18} \mathrm{~F}$-FDG PET/CT showed an increase in size and uptake in the lung lesion and a further increase in serum thyroglobulin level $(135.7 \mathrm{ng} / \mathrm{mL})$. Although the patient was clinically asymptomatic, ${ }^{68} \mathrm{Ga}$-DOTATATE PET/CT showed uptake in the axillary lymph nodes and lung lesions (Fig. 2), and PRRT was planned. 
FIGURE 2. Whole-body ${ }^{131}$ d diagnostic scintigraphy scan (A), ${ }^{18} \mathrm{~F}-\mathrm{FDG} \mathrm{PET} / \mathrm{CT}$ maximum-intensity-projection scan (B), ${ }^{68} \mathrm{Ga}-\mathrm{DOTATATE}$ maximum-intensityprojection scan (C), and coronal fused image (D) in known case of follicular variant of PTC. No abnormal tracer activity is seen in thoracic region on 131I scan. ${ }^{18} \mathrm{~F}-\mathrm{FDG}$-avid lesion and somatostatin receptor-avid lesion (on ${ }^{68} \mathrm{Ga}$-DOTATATE scan) are seen in thoracic region and axillary nodes. Arrows indicate tracer uptake or lack

thereof. A color version of this figure is available as a supplemental file at http://tech.snmjournals.org.

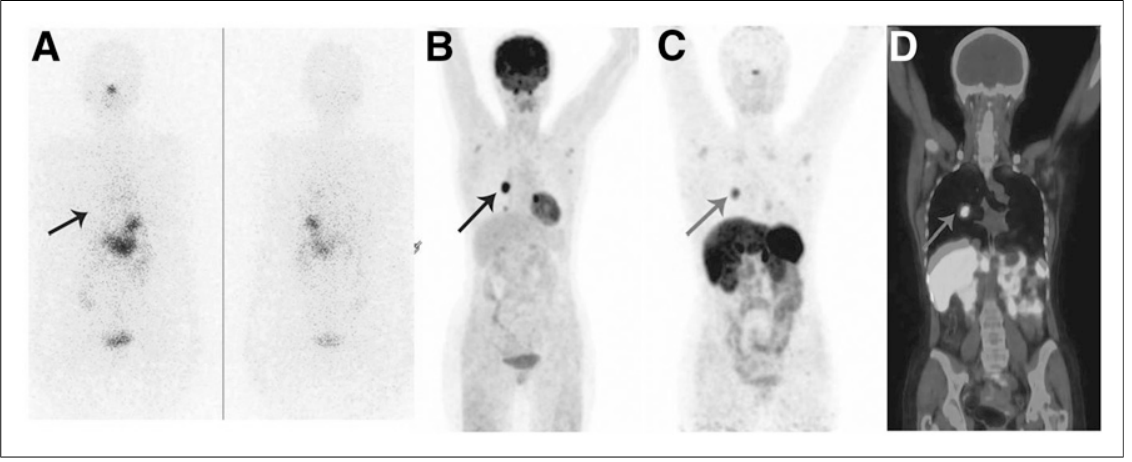

\section{Management Rationale}

In this case of TENIS syndrome, the disease burden was relatively low and the patient was asymptomatic, though the 6-mo follow-up ${ }^{18}$ F-FDG PET/CT showed increased uptake in the lesions. Empiric ${ }^{131} \mathrm{I}$ was of no benefit, and no abnormal disease focus was noted on the posttreatment scan. She was counseled on the available options and opted for PRRT.

Czepczyński et al. (5) undertook a retrospective analysis of 11 patients with TENIS syndrome who received PRRT with 4 doses of ${ }^{90}$ Y-DOTATOC. The RECIST criteria and thyroglobulin value were used for response evaluation. Median survival was 21 mo from the first course of PRRT. Only minor and transient hematologic toxicity in some patients was observed. The authors concluded that PRRT is generally well tolerated and may be a valuable option for some patients with ${ }^{131}$ I-refractory DTC. In our own study (6), we observed avid uptake on ${ }^{68} \mathrm{Ga}$-DOTATATE PET/CT and ${ }^{99 m}$ Tc-HYNIC-TOC scintigraphy in non-131 I-concentrating metastatic DTC in relatively few patients. In that series, 3 of 19 patients had an uptake score high enough (i.e., grade III or IV) to favor the feasibility of ${ }^{177} \mathrm{Lu}$-DOTATATE therapy. Also, interestingly, the chromogranin A level was increased in 3 patients with intense tracer uptake, suggesting that a possible neuroendocrine differentiation in the affected tissues led to the expression of chromogranin A with somatostatin receptor-avid expression in non- ${ }^{131}$ I-avid cases of DTC.
PRRT is expected to become a possible treatment option after ${ }^{131}$ I therapy for TENIS syndrome, but data on the efficacy of PRRT for DTC are thought insufficient and further investigation is warranted.

\section{TENIS SYNDROME WITH ${ }^{18}$ F-FDG-AVID LESIONS THAT ARE MINIMALLY OR NON-68GA-DOTATATE-AVID}

\section{Case 3}

A 55-y-old man (Fig. 3) underwent total thyroidectomy, central compartment clearance, and right modified neck dissection, with histopathologic findings suggestive of PTC with lymph node metastasis. Posttreatment scanning showed only a neck focus. Four years afterward, he presented with left supraclavicular lymphadenopathy: fine-needle aspiration of the lymph node demonstrated metastatic PTC. Intense ${ }^{18} \mathrm{~F}-\mathrm{FDG}$ uptake was seen in multiple cervical, supraclavicular, and mediastinal lymph nodes and in lung nodules, whereas scanning was negative for ${ }^{131}$ I uptake. A trial of redifferentiation therapy with retinoic acid was unsuccessful, with negative results seen on ${ }^{131}$ I scanning, and the serum stimulated thyroglobulin level at that time was $273 \mathrm{ng} / \mathrm{mL} .{ }^{68} \mathrm{Ga}$-DOTATATE PET/CT demonstrated faint uptake in the ${ }^{18} \mathrm{~F}-\mathrm{FDG}$-avid lesions; hence PRRT was not an option. The patient is currently being followed up with ${ }^{18} \mathrm{~F}-\mathrm{FDG}$ PET/CT and will be considered for tyrosine kinase inhibitors should the metastatic lesions demonstrate a rapid increase in uptake.
FIGURE 3. Whole-body ${ }^{131}$ I diagnostic scintigraphy scan (A), ${ }^{18} \mathrm{~F}-\mathrm{FDG}$ PET/CT maximum-intensity-projection scan and coronal fused image (B), and ${ }^{68} \mathrm{Ga}-$ DOTATATE maximum-intensity-projection scan (C) of known case of PTC in patient with raised serum thyroglobulin level. No abnormal ${ }^{131}$ I activity is seen in thoracic or neck regions. Intense ${ }^{18} \mathrm{~F}-\mathrm{FDG}$ activity is seen in thoracic, left supraclavicular, and right upper parapharyngeal regions on ${ }^{18} \mathrm{~F}-\mathrm{FDG}$ PET/CT, whereas ${ }^{68} \mathrm{Ga}$-DOTATATE scan shows faint tracer activity in these regions, as compared with liver. Arrows indicate tracer uptake or lack thereof. A color version of this figure is available as a supplemental file at http://tech.snmjournals.org. 


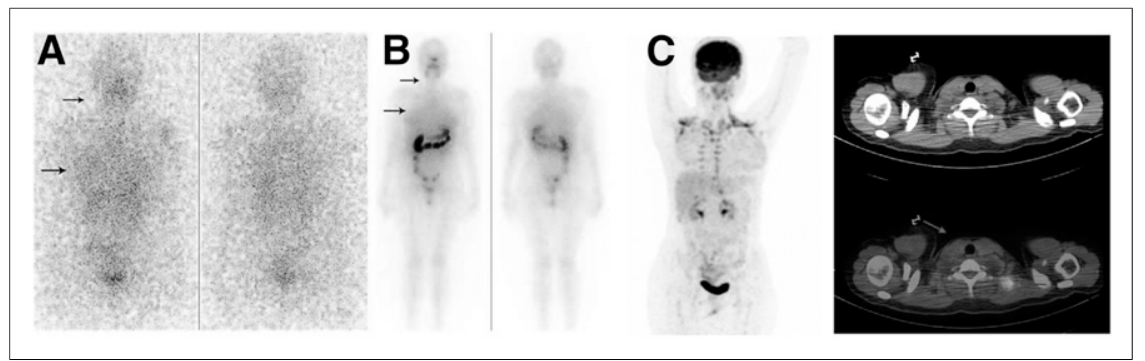

FIGURE 4. Whole-body ${ }^{131}$ I diagnostic scintigraphy scan (A), posttherapy (131/ ablation) scintigraphy scan (B), and ${ }^{18} \mathrm{~F}-\mathrm{FDG}$ PET/CT scan (C) of known case of PTC with raised serum thyroglobulin level. No abnormal ${ }^{131}$ I activity in neck or thoracic regions is seen on diagnostic or posttherapy scan. ${ }^{18} \mathrm{~F}-\mathrm{FDG}$ uptake is seen only in brown fat of neck (lower arrow in C).

Arrows indicate tracer uptake or lack thereof. A color version of this figure is available as a supplemental file at http:// tech.snmjournals.org.

\section{Management Rationale}

In a patient with TENIS syndrome, the presence of largevolume, rapidly progressing symptomatic disease (the definition criteria of which have yet to be finalized) is an indication for considering oral tyrosine kinase inhibitors. Our experience with redifferentiation therapy using retinoic acid has not been such as to allow us to recommend its routine use in patients with TENIS syndrome.

For patients with painful non- ${ }^{131} \mathrm{I}$-concentrating skeletal metastases, external-beam radiotherapy is a frequently considered option in practice, though it is not suited for patients with pulmonary metastases. Romesser et al. (7) studied 66 patients with gross residual or unresectable nonanaplastic nonmedullary thyroid cancer who were treated with external-beam radiotherapy. They concluded that external-beam radiotherapy is safe and effective, with greater than $85 \%$ locoregional control in patients with nonmetastatic disease and $90 \%$ locoregional control in patients treated with concurrent chemotherapy. Similarly, Sun et al. (8) suggested adoption of this approach in patients who are elderly or have massive primary disease, extensive extracapsular spread, or macroscopic ${ }^{131}$ I-negative components. They concluded that intensity-modulated radiation therapy offers clear dosimetric advantages with regard to tumor coverage and sparing of organs such as the larynx, thus reducing late toxicities to less than $5 \%$.

\section{TENIS SYNDROME WITH NORMAL ${ }^{18}$ F-FDG PET/CT FINDINGS}

\section{Case 4}

A 22-y-old woman (Fig. 4) with PTC with lymph node metastasis, who had previously undergone total thyroidectomy with nodal dissection and ${ }^{131} \mathrm{I}$ ablation, presented with an elevated level of serum thyroglobulin (stimulated thyroglobulin, $55 \mathrm{ng} / \mathrm{mL}$ ) but ${ }^{131} \mathrm{I}$ scintigraphy negative for uptake. Neck ultrasonography showed normal findings, and whole-body ${ }^{18} \mathrm{~F}-\mathrm{FDG}$ PET/CT was negative for any anatomic disease. A decision was made to observe the patient and follow up annually with neck ultrasonography and ${ }^{18} \mathrm{~F}-\mathrm{FDG}$ PET/CT.

\section{Management Rationale}

In our experience, negative ${ }^{18} \mathrm{~F}-\mathrm{FDG}$ PET results in the setting of an elevated serum thyroglobulin level forecasts a favorable disease prognosis and long progression-free survival.

One needs to look critically at the posttreatment scan before designating a case as TENIS syndrome. Not infrequently, when ${ }^{131} \mathrm{I}$ is used for the diagnostic scan, a small focus may be missed that becomes obvious on the posttreatment scan (4).

\section{PREDICTION OF AGGRESSION IN A PARTICULAR LESION BY DUAL-TRACER IMAGING}

\section{Case 5}

A 56-y-old man with PTC had undergone total thyroidectomy and nodal dissection at the time of diagnosis, followed by ${ }^{131}$ I ablation. He later presented with a serum thyroglobulin level of $205 \mathrm{ng} / \mathrm{mL}$, which increased to $300 \mathrm{ng} / \mathrm{mL}$ after TSH stimulation during follow-up, whereas diagnostic ${ }^{131}$ I whole-body scanning was negative for any obvious disease focus. ${ }^{18} \mathrm{~F}-\mathrm{FDG}$ PET/CT (noncontrast) showed a tiny nodule in the left lung (Fig. 5): he underwent empiric ${ }^{131}$ I therapy, and the posttreatment ${ }^{131} \mathrm{I}$ scan showed tracer activity in the left thoracic region corresponding to the lesion depicted on ${ }^{18}$ F-FDG PET/CT (Fig. 5).

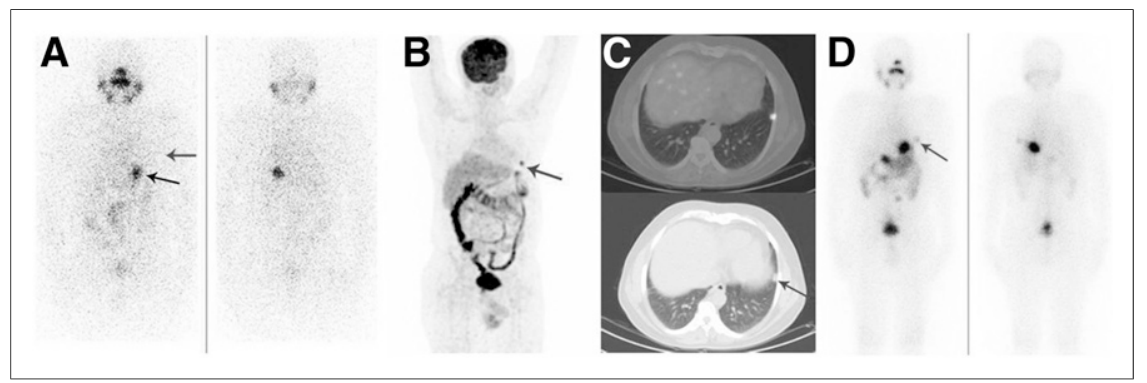

${ }^{18}$ F-FDG activity is seen in left lung nodule, and posttherapy ${ }^{131}$ I scan shows tracer activity in left thoracic region. Arrows indicate tracer uptake or lack thereof. A color version of this figure is available as a supplemental file at http://tech.snmjournals.org.

FIGURE 5. Whole-body ${ }^{131}$ I diagnostic scintigraphy scan (A), ${ }^{18} \mathrm{~F}-\mathrm{FDG} \mathrm{PET} / \mathrm{CT}$ maximum-intensity-projection scan (B), axial PET/CT and CT images (C), and posttherapy $\left({ }^{131} \mathrm{I}\right.$ ablation) whole-body scintigraphy scan (D) of known case of PTC with raised serum thyroglobulin level. Diagnostic ${ }^{131}$ I scan show physiologic tracer activity in stomach and no abnormal tracer activity in thoracic region, whereas 

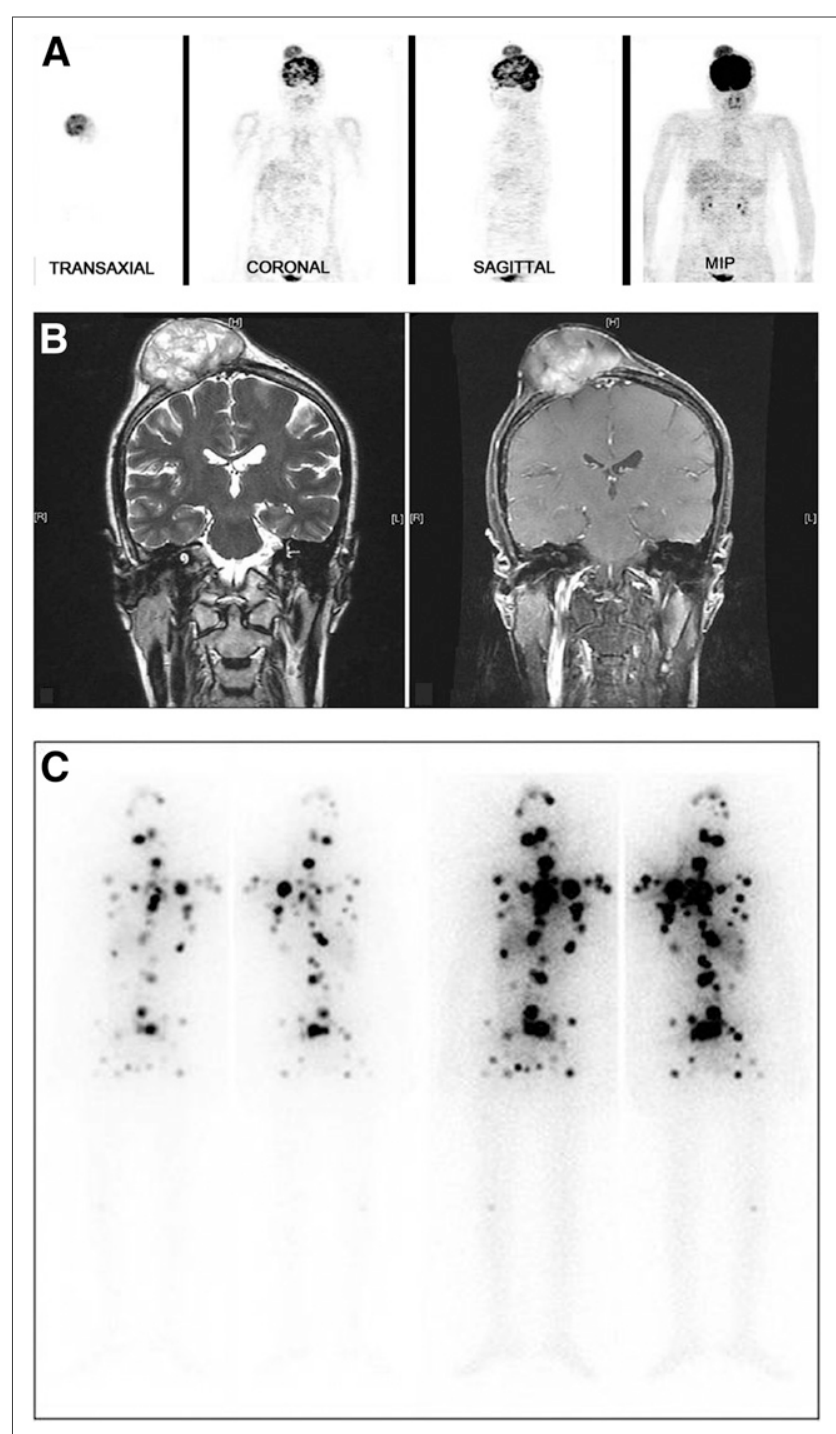

FIGURE 6. (A) Whole-body ${ }^{18} \mathrm{~F}-\mathrm{FDG}$ PET scans demonstrate no uptake in any skeletal metastatic lesion. Uptake is seen only in scalp lesion (for which patient presented to clinic). (B) Coronal T2-weighted (left) and postcontrast (right) MR images demonstrate hyperintense (on T2-weighted image), well-defined, invasive, heterogeneously enhancing soft-tissue lesion involving both tables of skull and extending to dural lining but remaining extraaxial. (C) Posttreatment ${ }^{131}$ I scan just before patient was discharged after second dose of ${ }^{131} \mid$ demonstrates extensive skeletal metastases concentrating ${ }^{131}$ I avidly. Incidentally noted is relatively low uptake in scalp lesion. (Reproduced with permission of (11).) MIP = maximum-intensity projection.

Typically, a flip-flop has been described between ${ }^{131}$ I scanning and ${ }^{18} \mathrm{~F}$-FDG PET with regard to DTC metastases, though the feasibility of concurrent ${ }^{18} \mathrm{~F}$-FDG and ${ }^{131} \mathrm{I}$ uptake has been reported, with avid ${ }^{18} \mathrm{~F}$-FDG uptake in metastatic lesions being postulated in recent years to be an important adverse prognostic factor $(9-11)$. Routine use of ${ }^{18} \mathrm{~F}-\mathrm{FDG}$ PET/CT in ${ }^{131} \mathrm{I}-$ concentrating metastatic DTC is not advocated at present. However, a dual-tracer evaluation (with ${ }^{131} \mathrm{I}$ and ${ }^{18} \mathrm{~F}-\mathrm{FDG}$ ) can provide valuable information on the future course of a particular lesion.

\section{Management Rationale}

The prognostic value of ${ }^{18} \mathrm{~F}-\mathrm{FDG}$ PET for DTC is often controversial, but intense ${ }^{18} \mathrm{~F}$-FDG uptake is generally regarded as an indicator of progression. Also, the advantage of ${ }^{18} \mathrm{~F}-\mathrm{FDG}$ PET, compared with other risk factors, is its capability of assessing lesion heterogeneity.

\section{Case 6}

A 66-y-old woman with DTC and extensive skeletal metastases throughout the body presented with progressively increasing scalp lesion (Fig. 6) and had been treated with ${ }^{131}$ I therapy twice previously. Whole-body ${ }^{18} \mathrm{~F}$-FDG PET (Fig. 6A), in contrast to ${ }^{131}$ I scanning, demonstrated a solitary focus of abnormal uptake corresponding to the scalp lesion but no uptake in any of the other skeletal metastatic disease. Interestingly, a review of the previous posttreatment scan showed relatively low uptake in the scalp lesion, compared with the other lesions, suggesting this particular metastasis to have heterogeneous behavior compared with the rest.

\section{Management Rationale}

Heterogeneous behavior by one or a few metastatic lesions compared with the rest is possible, and high ${ }^{18} \mathrm{~F}$ FDG uptake suggests that such lesions have an aggressive biology. Special emphasis needs to be given to managing these lesions with other modalities, as they will likely give rise to ${ }^{131}$ I-refractory clones in due course. A neurosurgical opinion was sought for the particular progressively increasing lesion in this case, but surgery was denied in view of the widespread metastases.

\section{${ }^{18}$ F-FDG UPTAKE AS AN OUTCOME DETERMINANT IN 131I-AVID LESIONS \\ Case 7}

A 56-y-old man with the follicular variant of PTC and metastases in the lungs and mediastinal nodes had undergone total thyroidectomy and lymph node dissection. He received multiple therapeutic doses of ${ }^{131} \mathrm{I}$ up to a cumulative dose of $37 \mathrm{GBq}(1 \mathrm{Ci})$, though the disease persisted and the stimulated thyroglobulin level was $300 \mathrm{ng} / \mathrm{mL} .{ }^{18} \mathrm{~F}-\mathrm{FDG}$ PET/CT demonstrated avid foci in all mentioned metastases (Fig. 7), possibly indicating ${ }^{131} \mathrm{I}$ refractoriness.

\section{Case 8}

A 51-y-old man with the follicular variant of PTC and metastases in the lymph nodes, skeleton, and lung had undergone total thyroidectomy with bilateral neck dissection. The patient was treated with $9,250 \mathrm{MBq}(250 \mathrm{mCi})$ of ${ }^{131} \mathrm{I}$, with imaging demonstrating ${ }^{131} \mathrm{I}$ uptake in the neck, lung lesions, and rib lesion and no abnormal ${ }^{18}$ F-FDG uptake. A 6-mo follow-up whole-body ${ }^{131}$ I scan was negative for any disease, and the level of stimulated serum thyroglobulin decreased from 200 to $0.26 \mathrm{ng} / \mathrm{mL}$ (Fig. 8). The negative ${ }^{18} \mathrm{~F}-$ FDG PET/CT results in this patient (Fig. 8C) were commensurate with the excellent response and favorable outcome. 


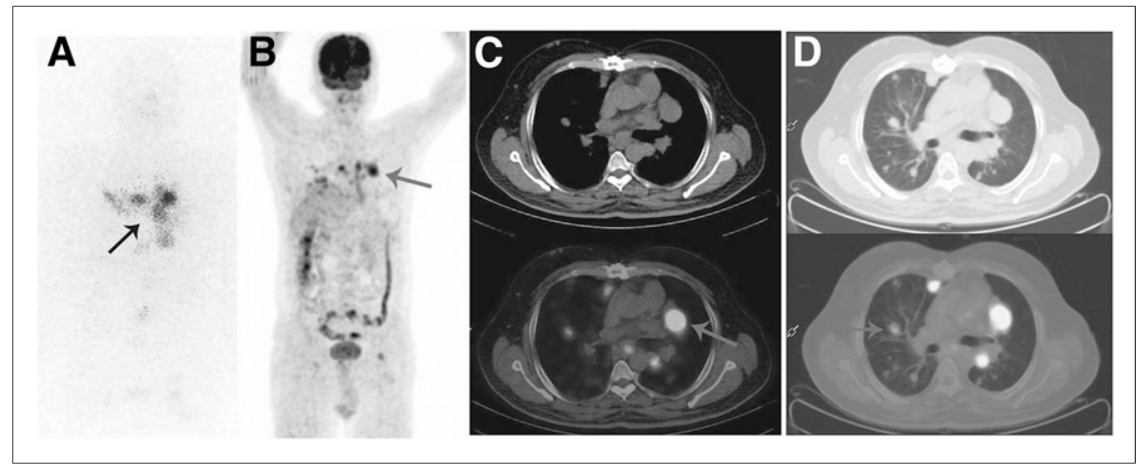

FIGURE 7. Posttherapy ( ${ }^{131} \mid$ ablation) whole-body scintigraphy scan $(A)$, whole-body ${ }^{18} \mathrm{~F}-\mathrm{FDG} \mathrm{PET} / \mathrm{CT}$ maximumintensity-projection scan (B), and 2 levels of axial thoracic ${ }^{18} \mathrm{~F}$-FDG PET/CT and CT images ( $C$ and $D$ ) of known case of follicular variant of PTC in patient with raised serum thyroglobulin level who had received total cumulative dose of $37 \mathrm{GBq}(1 \mathrm{Ci})$ of ${ }^{131} \mathrm{I}$ at time of imaging. Persistent tracer activity is seen in thoracic region ( $A$ and $B$ ), and ${ }^{18} \mathrm{~F}-\mathrm{FDG}$ uptake is seen in mediastinal lymph nodes (C) and lung nodules (D). Arrows indicate tracer uptake or lack thereof. A color version of this figure is available as a supplemental file at http://tech.snmjournals.org.

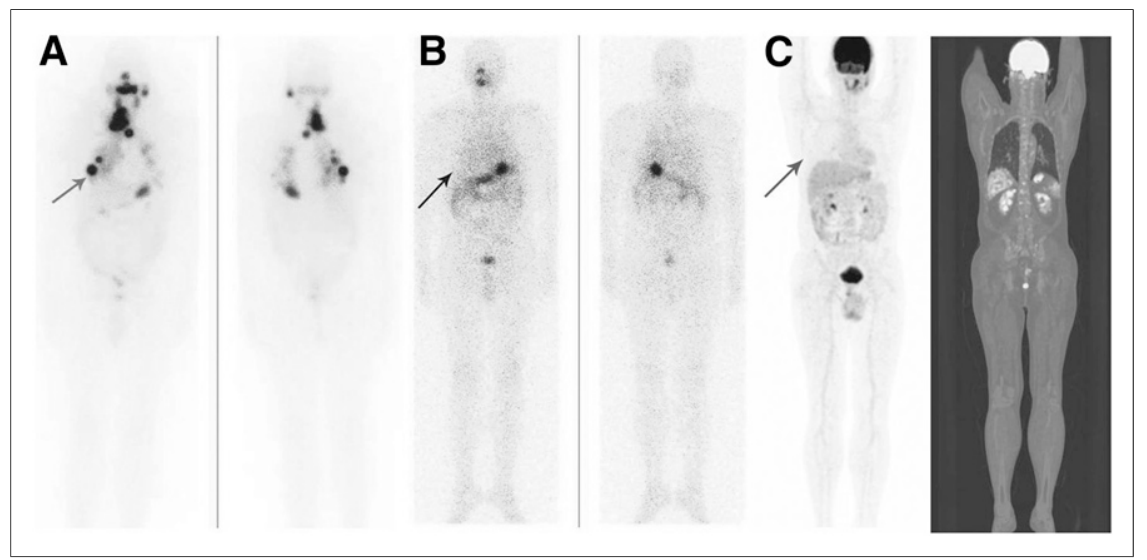

FIGURE 8. (A) Whole-body posttherapy (single fraction of ${ }^{131}$ I ablation) scintigraphy scan of DTC patient shows abnormal uptake in lung, solitary rib metastasis (posterior view), and neck. Whole-body posttherapy ${ }^{18} \mathrm{~F}-\mathrm{FDG}$ PET/CT scan (C) shows no abnormal tracer activity in thoracic or neck region. (B) Subsequent follow-up ${ }^{131}$ I scintigraphy scan shows excellent response. Arrows indicate tracer uptake or lack thereof. A color version of this figure is available as a supplemental file at http://tech.snmjournals.org.

\section{CONCLUSION}

The therapies for metastatic thyroid carcinoma and TENIS syndrome currently provided in the field are far from ideal, with various approaches being suggested, including those by our group (4). We have reported here on a gamut of representative cases in which molecular functional imaging was used to characterize metastatic lesions, group them into classes, and apply the best treatment for each class - this being the basis for individualization of treatment regimens. We believe that development of a clinical algorithm for the systematic stratification and individualized treatment of metastatic thyroid carcinoma and TENIS syndrome would be of great value to patient management.

\section{DISCLOSURE}

No potential conflict of interest relevant to this article was reported.

\section{REFERENCES}

1. Silberstein EB. The problem of the patient with thyroglobulin elevation but negative iodine scintigraphy: the TENIS syndrome. Semin Nucl Med. 2011;41:113-120.

2. Ma C, Xie J, Kuang A. Is empiric ${ }^{131} \mathrm{I}$ therapy justified for patients with positive thyroglobulin and negative ${ }^{131} \mathrm{I}$ whole-body scanning results? J Nucl Med. 2005;46:1164-1170.
3. Mazzaferri EL. Empirically treating high serum thyroglobulin levels. $J$ Nucl Med. 2005;46:1079-1088.

4. Basu S, Dandekar M, Joshi A, D'Cruz A. Defining a rational step-care algorithm for managing thyroid carcinoma patients with elevated thyroglobulin and negative on radioiodine scintigraphy (TENIS): considerations and challenges towards developing an appropriate roadmap. Eur J Nucl Med Mol Imaging. 2015;42:1167-1171.

5. Czepczyński R, Matysiak-Grześ M, Gryczyńska M, et al. Peptide receptor radionuclide therapy of differentiated thyroid cancer: efficacy and toxicity. Arch Immunol Ther Exp (Warsz). 2015;63:147-154.

6. Jois B, Asopa R, Basu S. Somatostatin receptor imaging in non- ${ }^{131} \mathrm{I}$-avid metastatic differentiated thyroid carcinoma for determining the feasibility of peptide receptor radionuclide therapy with ${ }^{177} \mathrm{Lu}$-DOTATATE: low fraction of patients suitable for peptide receptor radionuclide therapy and evidence of chromogranin A level-positive neuroendocrine differentiation. Clin Nucl Med. 2014;39:505-510.

7. Romesser PB, Sherman EJ, Shaha AR, et al. External beam radiotherapy with or without concurrent chemotherapy in advanced or recurrent non-anaplastic non-medullary thyroid cancer. J Surg Oncol. 2014;110:375-382.

8. Sun XS, Sun SR, Guevara N, et al. Indications of external beam radiation therapy in non-anaplastic thyroid cancer and impact of innovative radiation techniques. Crit Rev Oncol Hematol. 2013;86:52-68.

9. Salvatori M, Biondi B, Rufini V. Imaging in endocrinology: $2-\left[{ }^{18} \mathrm{~F}\right]-$ fluoro-2deoxy-D-glucose positron emission tomography/computed tomography in differentiated thyroid carcinoma: clinical indications and controversies in diagnosis and follow-up. Eur J Endocrinol. 2015;173:R115-R130.

10. Treglia G, Muoio B, Giovanella L, Salvatori M. The role of positron emission tomography and positron emission tomography/computed tomography in thyroid tumours: an overview. Eur Arch Otorhinolaryngol. 2013;270:1783-1787.

11. Basu S, Mahajan A. Discordant and aggressive tumor biology of solitary scalp metastasis amidst widespread skeletal metastases in differentiated thyroid carcinoma: functional radionuclide and MR imaging features and clinical correlates. Indian J Cancer. 2014;51:613-614. 